\title{
Bioquímica Mitocondrial e Estresse Oxidativo no Transtorno Bipolar: Novos Horizontes
}

\author{
Luiz Arthur Rangel Cyrino ${ }^{1}$, Daniela Delwing-de-Lima ${ }^{2}$, Oliver Matheus Ullmann ${ }^{1}$, \\ Thayna Patachini Maia ${ }^{1}$, Ana Carolina Sardo ${ }^{1}$
}

\begin{abstract}
RESUMO
Este artigo é uma revisão do efeito do estresse oxidativo (EO) no Transtorno Bipolar (TB). Esta doença é caracterizada como sendo crônica, grave, com alta morbidade, mortalidade e altas taxas de suicídio. É uma doença progressiva, com episódios cada vez mais curtos e frequentes ao longo do tempo. Modificações ocorrem no encéfalo, como alterações na neuroplasticidade, neurotransmissão, falhas na apoptose, ativação no processo imunoinflamatório, com alterações na via de sinalização do cálcio e, mais recentemente, sobre o EO. Esses eventos envolvem uma reorganização patológica no encéfalo e, portanto, estão associados a alterações morfológicas, tais como redução do volume do córtex pré-frontal, do hipocampo e da amígdala aumentada. Essas alterações estruturais e bioquímicas são conhecidas como neuroprogressão, e apresentam diferenças entre o estágio inicial e o final do TB. Neurônios e células da glia têm uma das mais altas demandas de energia, quando estas alterações bioquímicas e anatômicas afetam essas células e, principalmente, organelas, como as mitocôndrias. A mitocôndria é uma organela especializada que gera trifosfato de adenosina (ATP) sendo considerada a principal fonte de espécies reativas produzidas principalmente pela cadeia de transporte de elétrons (CTE), levando, assim, ao EO. O papel do EO na fisiopatologia da TB tem sido investigado em vários estudos, relatando mudanças nos níveis das enzimas antioxidantes, na peroxidação lipídica e do óxido nítrico (NO). Os mecanismos fisiopatológicos do TB contribuem para uma melhor compreensão da atividade da doença e podem revelar possíveis soluções para o diagnóstico e prognóstico.
\end{abstract}

Palavras-chave: Radicais livres. Metabolismo energético. Mitocôndrias. Transtorno bipolar. Estresse oxidative. Sistema nervoso central.

MITOCHONDRIAL BIOCHEMISTRY AND OXIDATIVE STRESS IN BIPOLAR DISORDER: NEW HORIZONS

\section{ABSTRACT}

This article is a review of the effect of oxidative stress on Bipolar Disorder (BD). This disease is characterized as being chronic, severe with high morbidity, mortality, and high suicide rates. It is a progressive disease with episodes becoming successively shorter and frequent over time. Multiple alterations occur in the brain such as changes in neuroplasticity, neurotransmission, failures in apoptosis, activation in the immune-inflammatory process, with changes in the calcium signaling pathway; and more recently, on oxidative stress. These events involve a pathological reorganization in the brain and therefore are associated with morphological changes such as, a reduction in the volume of the prefrontal cortex, hippocampus, and an enlarged amygdala. These structural and biochemical changes are known as neuroprogression, with a difference between the initial and final stages of BD. Neurons and glial cells have a high energy demand, where biochemical and anatomic changes affect these cells and mainly organelles such as mitochondria. Mitochondria is a specialized organelle that generates adenosine triphosphate. It is considered the primary source of reactive oxygen species, produced through the electron transport chain, leading to oxidative stress. The role of oxidative stress in the pathophysiology of BD has been investigated in several studies, and consistently reported changes in antioxidant enzymes, lipid peroxidation, and nitric oxide levels. Pathophysiological mechanisms of BD contributes to an improved understanding of disease activity and may reveal possible solutions for diagnosis and prognosis.

Keywords: Free radicals. Energy metabolism. Mitochondria. Bipolar disorder. Oxidative stress. Central nervous system.

RECEBIDO EM: 29/6/2019

MODIFICAÇÕES SOLICITADAS EM: 25/8/2019

ACEITO EM: 6/9/2019

\footnotetext{
Universidade da Região de Joinville - Univille. Joinville/SC, Brasil.

${ }_{2}^{2}$ Autora correspondente. Universidade da Região de Joinville - Univille. Rua Paulo Malschitzki, 10 - Zona Industrial Norte. Joinville/SC, Brasil. CEP $89219-710$. http://lattes.cnpq.br/5765728791389269. https://orcid.org/0000-0001-5335-5102. danidelwing@hotmail.com
} 


\section{INTRODUÇÃO}

O Transtorno Bipolar (TB) é uma doença crônica e grave que afeta aproximadamente $1,1 \%$ da população mundial e está associado a uma alta taxa de comorbidades clínicas. Sua fisiopatologia é complexa, multifatorial e não é totalmente compreendida, sendo influenciada, de forma consistente, por fatores genéticos e ambientais (CLEMENTE et al., 2015). Caracteriza-se por múltiplas alterações no encéfalo, entre as quais a neuroplasticidade, a cronobiologia, falhas na apoptose, o desenvolvimento cerebral alterado, a neurotransmissão e o processo imune-inflamatório, além da via de sinalização do cálcio, e, mais recentemente, o EO, com fortes alterações na bioenergética celular (SIGITOVA et al., 2017). A doença também inclui déficits cognitivos, resistência ao tratamento e alterações biológicas periféricas e em estruturas cerebrais, como atrofia cortical e dilatação dos ventrículos. Tais alterações tornam-se mais pronunciadas após múltiplos episódios de humor (mania ou depressão). Uma hipótese para explicar essas fortes mudanças do estado de humor a partir do dano cumulativo e patologias relacionadas ao TB, é conhecida como carga alostática (VIETA et al., 2013). Essa teoria busca entender como as mudanças cumulativas associadas às doenças psiquiátricas refletem-se na progressão da doença (KAPCZINSKI et al., 2008). Assim, a alostase é a capacidade de obter estabilidade por meio de alterações, o que permite ao organismo responder a várias situações mediante alterações fisiológicas. Se, no entanto, esses processos se tornarem extremos ou ineficientes, a responsividade do corpo diminui e os processos acumulam-se para que mais sistemas regulatórios sejam comprometidos (carga alostática), prejudicando a plasticidade neuronal e a neurotransmissão (MCEWEN, 2000). Esse conjunto de mudanças fisiológicas, que ocorre com o curso da doença, foi associado a outro conceito recentemente desenvolvido, chamado neuroprogressão (BERK et al., 2011). Dessa forma, este termo é utilizado para explicar a progressão dos distúrbios e a consequente aceleração dos mecanismos de doença (GRANDE et al., 2012; MCEWEN; GIANAROS, 2011). Neste contexto, com a progressão da doença, ocorre um aumento na frequência e gravidade dos episódios de mania e depressão ao longo dos anos, levando ao aumento no número de comorbidades médicas e psiquiátricas associadas, desequilíbrio entre fatores pró e anti-inflamatórios, redução das neurotrofinas e aumento do EO. Este processo intoxica o corpo, liberando compostos e produzindo um remodelamento patológico do encéfalo e pior funcionamento cognitivo e emocional, com diminuição da produtividade na vida pessoal e profissional e de recuperação clínica (GAMA et al., 2013). Assim, o TB pode ser visto como uma doença inflamatória multissistêmica, sendo representado por alterações em biomarcadores séricos (SYLVIA et al., 2015) que poderiam funcionar como indicadores de toxicidade celular nestes pacientes (ROWLAND et al., 2018). A análise de um conjunto de biomarcadores (marcadores inflamatórios, EO e neurotrofinas) mostrou que pacientes com TB de início agudo têm uma taxa de toxicidade sistêmica significativamente maior do que controles saudáveis, mas menor do que pacientes com sepse (PFAFFENSELLER et al., 2013). Desse modo, compreender os mecanismos fisiopatológicos sistêmicos que contribuem para a disfunção resultante de múltiplos episódios de humor do TB, e identificar as vias associadas aos mediadores alostáticos envolvidos na neuroproteção, EO e inflamação, podem ser importantes para melhorar nossa compreensão da atividade da doença. Os diferentes biomarcadores podem estar associados à atividade da doença e à neuroprogressão, indicando se a doença está ativa ou em remissão. Vale ressaltar que marcadores sistêmicos já foram implicados no TB como mediadores da alostase (JUSTER et al., 2013). Entre os mediadores da alostase e da neuroprogressão, destacam-se os glicocorticoides. Estes hormônios promovem adaptação dinâmica do organismo principalmente por meio da regulação da disponibilidade de compostos energéticos, entre outros efeitos. Quando em excesso e de forma crônica, podem causar disfunções em compartimentos celulares essenciais, tais como as mitocôndrias, produzindo um aumento do EO e de marcadores pró-inflamatórios, assim como a diminuição de fatores neurotróficos; processos esses já evidenciados em pacientes bipolares. Esta exposição excessiva aos glicocorticoides, observada no TB, acaba produzindo alterações neuroanatômicas, cognitivas e funcionais, que são mais pronunciadas em pacientes crônicos com múltiplos episódios, mas são, geralmente, sutis naqueles em estágios iniciais (BERK et al., 2011). Apesar da psiquiatria necessitar de biomarcadores confiáveis e acessíveis, que facilitem o diagnóstico de forma precoce, estes ainda são insuficientes e caem em aproximadamente três categorias promissoras: 1 - genética, 2 - descobertas de imagens e 3 - níveis de metabólitos de moléculas. Neste último grupo encontram-se dosagens periféricas de diversas substâncias oriundas de células neuronais e gliais, tais como: 1 - as neurotrofinas: Fator Neurotrófico Derivado do Cérebro (BDNF); Fator Neurotrófico Derivado da Glia (GDNF) e Fator de Crescimento Neuronal (NGF); 2 - as citocinas pró-inflamatórias: Interleucina-6 (IL6), Interleucina-1 (IL-1) e o Fator de Necrose Tumoral 
Alfa (TNF- $\alpha$ ), 3 - substâncias relacionadas ao EO: superóxido dismutase (SOD), catalase (CAT), glutationa peroxidase (GSH-Px), 3-nitrotirosina (3-NT), e as Substâncias Reativas ao Ácido Tiobarbitúrico (TBA-RS), e o último grupo: 4 - marcadores de disfunção do Ciclo de Krebs (CK), tais como citrato sintase, succinato desidrogenase e malato desidrogenase (GRANDE et al., 2014; DE SOUSA et al., 2015; SCAINI et al., 2016).

Todas essas alterações bioquímicas estudadas impactam diretamente no encéfalo e, principalmente, nas células neuronais e em organelas como as mitocôndrias. Os neurônios são células de alta demanda energética. Um único neurônio cortical em repouso consome aproximadamente 4,7 milhões de moléculas de ATP por segundo para processar várias funções biológicas. A taxa de utilização de ATP é três vezes maior na substância cinzenta do que na substância branca no cérebro humano (ZHU; LEE; CHEN, 2018). Isso torna os neurônios responsáveis por $20 \%$ a $25 \%$ de todo o oxigênio e glicose consumidos pelo organismo (MANSUR; BRIETZKE, 2012). Em algumas porções dos neurônios ocorre uma demanda maior de fornecimento de energia, tais como os terminais pré-sinápticos e pós-sinápticos que regulam a neurotransmissão, os cones ativos de crescimento ou ramos axonais que regulam a plasticidade sináptica de curto prazo e, finalmente, os nódulos de Ranvier (ZHANG et al., 2010). Para que estas mitocôndrias cheguem aos seus destinos, é necessário que ocorra o transporte mitocondrial neuronal. Este é bidirecional e de longo alcance, e depende dos microtúbulos, ocorrendo com uma velocidade média entre 0,32 e 0,91 $\mu \mathrm{m} / \mathrm{s}$ (MACASKILL; KITTLER, 2010). A maior parte da biogênese das mitocôndrias neuronais acontece principalmente no soma do neurônio, quando são, então, distribuídas ao longo do axônio. Os mecanismos que regulam o transporte direcional das mitocôndrias são baseados em receptores na membrana. Eles capturam a informação do potencial de membrana mitocondrial (PMM) por meio da concentração de íons ou substâncias, como $\mathrm{H}^{+}$, $\mathrm{Ca}^{2+}$, ATP, difostato de adenosina (ADP), piruvato e dinucleotídeo de nicotinamida e adenina (NADH). Após a sinalização, as vias de transdução são ativadas de diferentes formas. Uma vez formadas e transportadas, as mitocôndrias podem ficar ancoradas em certas regiões ou apresentar mudança de direção. Assim, para manter a plasticidade neuronal e a confiabilidade da neurotransmissão, é necessário ter algumas das mitocôndrias ancoradas no terminal pré-sináptico. Estas mitocôndrias fornecem uma quantidade estável e contínua de ATP. Cerca, no entanto, de $20 \%$ a $30 \%$ das mitocôndrias axonais são móveis (CHEN; SHENG,
2013). Ao passarem por esses terminais de forma rápida e temporária, estas fornecem ATP, alterando, assim, a energia sináptica e influenciando as atividades das mesmas. Nessa direção, Sun et al. (2013) demonstraram pela primeira vez que este é um dos principais mecanismos subjacentes à variação pré-sináptica, quando a falta de mitocôndrias no terminal axonal prejudica a neurotransmissão pela diminuição de ATP e, consequentemente, da força de liberação das vesículas sinápticas, assim como a capacidade de bloquear e tamponar o cálcio ionizado da terminação axônica (IVANNIKOV; SUGIMORI; LLINÁS, 2013). Quanto à região pós-sináptica, pesquisas realizadas com métodos proteômicos, baseados em espectrometria de massa (FÖCKING et al., 2016), observaram proteínas do citoesqueleto facilitando o movimento e a sinalização de alta densidade de receptores e complexos ativados que são críticos para a neurotransmissão normal e plasticidade sináptica. Desta forma, sabemos que a plasticidade sináptica é altamente dependente da função mitocondrial e do metabolismo energético. Quando as mitocôndrias alteram sua motilidade ou distribuição, ou quando sua integridade é prejudicada sob certas condições de estresse fisiopatológico, estas podem ser implicadas na patogênese de vários distúrbios neuropsiquiátricos importantes, como no TB e na esquizofrenia (CHU, 2019). Outras células fortemente envolvidas no processo da neuroprogressão são as células microgliais. Elas fazem contato íntimo com as sinapses, produzindo a degeneração sináptica, como nas doenças neurodegenerativas (HONG; DISSING-OLESEN; STEVENS, 2016). Estas células também estão relacionadas com a formação, proliferação e diferenciação de circuitos neurais, e ainda com a poda de conexões sinápticas em redes neurais. Quando esta comunicação é interrompida, pode-se observar efeitos devastadores na memória, sociabilidade, ansiedade e outros domínios comportamentais (WU et al., 2014). Graças às recentes pesquisas desenvolvidas por Herz et al. (2017), sabemos hoje que células microgliais e macrófagos não parenquimais, que se apresentam nas meninges, espaços perivasculares e plexo coroide, representam duas populações mieloides ontogeneticamente distintas. As células da microglia aparecem precocemente durante o desenvolvimento embrionário derivado do saco vitelino (E) 8.5 (GOMEZ et al., 2015), semeando o tecido cerebral antes do nascimento e permanecendo com uma população estável até o envelhecimento. Todos os outros macrófagos do sistema nervoso central (SNC) provavelmente originam-se de monócitos derivados da medula óssea. $\mathrm{O}$ volume de reposição da microglia é rápido, permitindo a renovação várias vezes ao longo da vida, a partir 
de processos associados à proliferação e apoptose (ASKEW et al., 2017). Apesar das origens distintas, microglia e macrófagos, que derivaram de monócitos, têm morfologias e funções fagocíticas semelhantes, contudo existe um subconjunto de funções exclusivas da microglia (GINHOUX; GUILLIAMS, 2016; PRINZ; PRILLER, 2017). Como são células imunes inatas do encéfalo, elas estão, de fato, procurando continuamente sinais que indiquem potenciais ameaças ao SNC, mesmo quando estas se encontram em seu estado de repouso (inativado) (HELLWIG; HEINRICH; BI$B E R, 2013)$. A presença de sinais químicos de infecções, traumas, substâncias endógenas e exógenas, ou a perda de sinais constitutivos anti-inflamatórios, podem ativar múltiplas vias de respostas. Estudos mostraram que a microglia e os macrófagos podem ser ativados em dois fenótipos significativos: classicamente ativados (M1) e alternativamente ativados (M2) (GEISSMANN et al., 2010). Esses diferentes fenótipos reativos, constituídos por transcrição e perfis não transcricionais, são caracterizados por liberação de citocinas, quimiocinas e fagocitose. No estágio inicial da doença, os sintomas podem ser seguidos pela polarização microglial M1, induzida por hiperativação neuronal (NAKAGAWA; CHIBA, 2014; GINHOUX et al., 2016). O fenótipo $M 1$ pode produzir citocinas pró-inflamatórias e metabólitos oxidativos, como o TNF- $\alpha$, IL-6, IL-1 $\beta$ e NO, fatores que podem causar danos adicionais e remover fibras nervosas danificadas pela fagocitose. A polarização microglial M2 anti-inflamatória, entretanto, é ativada em resposta a IL-4, IL-13 ou IL-10 (NGUYEN et al., 2011), que regula negativamente a função microglial M1, restaurando o tecido da homeostase, consequentemente atenuando os sintomas, suprimindo a inflamação, reparando o tecido e promovendo a cicatrização de feridas (KAWABORI; YENARI, 2014). Se a polarização $M 2$ é insuficiente, as funções microgliais M1 são mantidas e induzem disfunções da rede neural continuamente. A gravidade dos sintomas pode aumentar gradualmente de acordo com a frequência da polarização M1 (NAKAGAWA; CHIBA, 2014). Essas células poderiam manter mudanças sutis, como na atividade transcricional, o que afetaria sua sensibilidade a sinais anti-inflamatórios ou alteraria respostas a estímulos subsequentes, podendo até levar à morte de neurônios. É importante lembrar que a microglia ativada sempre esteve associada a efeitos neurotóxicos, e esse mecanismo está relacionado com a neuroprogressão, em que a ativação microglial é desregulada e a inflamação induzida pela microglia é intensa, ocorrendo efeitos diretos sobre os neurônios. Estes mecanismos são observados em todas as patologias no encéfalo, contribuindo para a progressão das doenças mentais, quando dano tecidual ou estresse celular produzem sinais de ativação microglial (KETTENMANN et al., 2011). Indivíduos com TB nos distintos estágios da doença (precoce e tardia) e nas diferentes fases (depressiva, maníaca e eutímica), apresentam alterações de alguns destes mediadores no sangue periférico. Assim, a neuroprogressão, a partir do remodelamento da estrutura cerebral, associada a essas alterações químicas, tem importantes implicações clínicas, posto que estágios iniciais e tardios da doença parecem apresentar diferentes características biológicas e, assim, possivelmente requerem diferentes estratégias de tratamento (FERENSZTAJN; RYBAKOWSKI, 2012). Os mecanismos que levam à diminuição da resistência orgânica em situações de estresse nos episódios agudos no TB, provavelmente envolvem vias de sinalização celular e organelas, por exemplo as mitocôndrias e retículo endoplasmático, que afetariam as células neuronais e gliais. Evidências sugerem um papel importante da disfunção mitocondrial no $T B$, produzindo um metabolismo energético alterado, mudanças nas enzimas da Cadeia Transportadora de Elétrons (CTE) da cadeia respiratória, níveis alterados do cálcio citoplasmático e alterações na ativação de genes mitocôndrias. O influxo de cálcio do citosol controla a taxa de produção mitocondrial de ATP. O cálcio intramitocondrial ativa três desidrogenases acopladas ao CK, que são o piruvato desidrogenase, isocitrato desidrogenase e $\alpha$-cetoglutarato desidrogenase. Além disso, a captação de cálcio modula o perfil espacial e temporal da sinalização intracelular de cálcio, regulando a motilidade e a morfologia mitocondrial, podendo levar à morte celular (HOPPE, 2010). Alterações nas concentrações de lactato também são significativamente mais altas em pacientes com TB do que os controles, o que indica o aumento do metabolismo mitocondrial e a utilização anaeróbica da glicose, consistente com o metabolismo mitocondrial alterado no TB. Assim, pesquisas têm contribuído para a compreensão destes mecanismos, explicando parcialmente a toxicidade relacionada com episódios de humor cumulativos no TB, considerando que esta revisão busca, principalmente, explicar alguns destes mecanismos a partir do EO no TB e sua relação com as alterações bioquímicas subjacentes à mitocôndria.

\section{METODOLOGIA}

As informações coletadas para essa revisão foram pesquisadas nas seguintes bases de dados disponíveis: ScienceDirect, PubMed/Medline, Embase e Google Scholar, Scopus e SciELO, levando-se em con- 
sideração apenas artigos nos idiomas inglês e português. Foram utilizados os seguintes termos para pesquisa: transtorno bipolar, estresse oxidativo, EROs, ERNs, radicais livres ( $R L)$, antioxidante, NO, peroxidação lipídica, TBA-RS, proteína carbonil 3-nitrotirosina, catalase, glutationa, oxidação do DNA e neuroprogressão. As publicações foram selecionadas por título e conteúdo no resumo inicialmente, e, depois, por conteúdo completo. Como critério de seleção foram usados os artigos a partir de 2000 até 2019 , com exceção de trabalhos clássicos, priorizando os artigos dos últimos cinco anos.

\section{MITOCÔNDRIA}

Mitocôndrias são organelas especializadas, encontradas somente em células eucariontes, sendo responsáveis por várias funções celulares, como modulação da atividade neuronal, morfogênese, neuroplasticidade e produção energética, sendo cruciais na morte celular. No geral, são compostas por dois complexos de membranas, separados por um espaço intermembrana, o qual possui inúmeras invaginações que formam as cristas mitocondriais, em que estão presentes as proteínas da CTE, cujo número e morfologia refletem a demanda de energia (DING et al., 2012). A síntese de proteínas mitocondriais envolve a expressão do DNA nuclear (nDNA) e do mtDNA, dando origem ao complexo mitocondrial (WIEDEMANN; PFANNER, 2017). O nDNA codifica a vasta maioria de proteínas mitocondriais, sendo responsável por sintetizá-las no citosol e importar para a membrana interna e externa da mitocôndria por meio da translocação de proteínas (KANG; FIELDEN; STOJANOVSKI, 2018). O mtDNA é composto por uma cadeia pesada e uma cadeia leve, contendo 28 genes e 9 genes, respectivamente (HÄLLBERG; LARSSON, 2014). Esses 37 genes são responsáveis pela síntese RNAs, sendo 2 genes responsáveis pelo RNA ribossômico, 22 genes responsáveis pelos RNAs transportadores e 13 pelos RNAs mensageiros que, após translação, geram polipeptídeos que estão envolvidos na fosforilação oxidativa (Oxphos) (BJÖRKHOLM et al., 2015). As proteínas geradas pelo mtDNA estão presentes em quatro das cinco proteínas da CTE, posto que mudanças em sua estrutura podem afetar o metabolismo. Conforme demonstrado na Figura 1, as mitocôndrias metabolizam carboidratos e ácidos graxos, produzindo energia por intermédio da geração de ATP. Essa geração ocorre na matriz mitocondrial, enquanto as etapas iniciais do metabolismo de carboidratos acontecem fora da organela e, para que isso ocorra, dois processos são necessários: CK e Oxphos. Inicialmente, a glicose é convertida em piruvato e, então, transportada para a matriz. Já os ácidos graxos, no entanto, são oxidados no interior da matriz mitocondrial. O ATP é produzido mediante três etapas interconectadas. Na primeira, o piruvato e os ácidos graxos são convertidos em moléculas conhecidas como acetil-CoA por meio de enzimas presentes na matriz. Na segunda etapa, essa substância torna-se a base para a cadeia de reações químicas conhecida como CK (PEI; WALLACE; 2018). Por fim, o ciclo é formado por uma série de reações químicas em que o dióxido de carbono e duas moléculas, ricas em elétrons, o NADH e o dinucleotídeo de flavina e adenina $\left(\mathrm{FADH}_{2}\right)$, são produzidos. Na terceira etapa, o NADH e FADH 2 são movidos para o interior da membrana mitocondrial. Na última etapa química, ocorre a doação de elétrons para a CTE, o que leva à produção de ATP. Para a execução desse processo, o complexo I e o II recebem elétrons do NADH e $\mathrm{FADH}_{2}$, respectivamente (ENRÍQUEZ, 2016). Ambos transferem esses para a Coenzima Q10, que é uma enzima essencial em virtude das suas propriedades antioxidantes. A Coenzima Q10 transfere os elétrons para o complexo III, quando são transportados para o complexo IV pelo Citocromo C (ALVAREZ-PAGGI et al., 2017). No complexo IV, elétrons são transferidos para o oxigênio molecular formando água (MILENKOVIC et al., 2017; ENRÍQUEZ, 2016). Finalmente, o hidrogênio é transportado pelo complexo $V$, também chamado de ATP sintase, de volta à matriz mitocondrial, para a formação de ATP por meio do ADP e do fosfato inorgânico. O ATP alimenta várias funções da célula e do organismo como um todo (VIDYASAGAR, 2015). Podemos ver que todas as estruturas da CTE dependem de certos níveis de codificação nuclear e mitocondrial, com exceção do Complexo II, que é codificado exclusivamente por mtDNA. O complexo II tem especial importância no metabolismo energético, posto que este complexo possui dois domínios catalíticos especializados: um deles transfere os elétrons recebidos do $\mathrm{FADH}_{2}$ para a Coenzima Q10, e o outro converte succinato em fumarato - gerando uma molécula de $\mathrm{FADH}_{2}$, participando tanto no $\mathrm{CK}$ quanto na CTE.

Com o passar do tempo, as funções mitocôndrias vão se tornando menos efetivas, o que inclui o desemparelhamento do complexo I e, com menor frequência, a diminuição da atividade do complexo III (SCAINI et al., 2016). A atividade alterada dos complexos da CTE leva à produção aumentada de EROs e ERNs (VALVASSORI et al., 2018), ao comprometimento do equilíbrio de cálcio intracelular e a mutações do DNA (MONICZEWSKI et al., 2015). Com isso, estas dis- 
Figura 1 - Produção energética pelas mitocôndrias

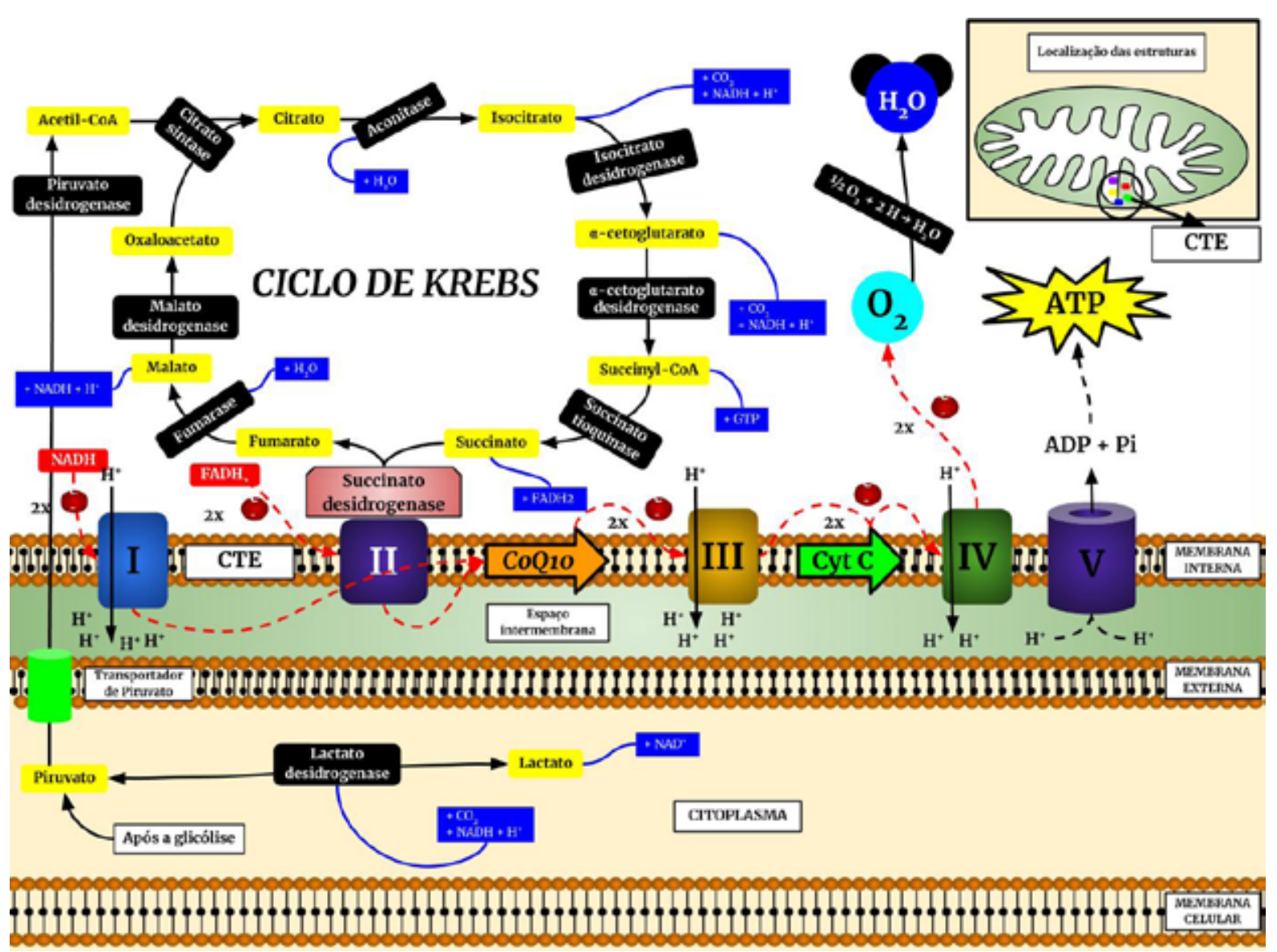

* Em organismos aeróbicos, a glicose é oxidada em $\mathrm{CO}_{2}$ e $\mathrm{H}_{2} \mathrm{O}$. O piruvato apresentado no citosol da célula é oxidado em acetil-CoA e pode, assim, entrar no $\mathrm{CK}$, que é composto de um complexo de enzimas, localizado no citosol mitocondrial de células eucarióticas. Com isso há a produção de $\mathrm{NADH}_{\text {e }}$ FADH ${ }_{2}$ que serão encaminhados para a CTE na membrana interna mitocondrial. O fluxo de elétrons ocorre por meio dos complexos proteicos, sendo acompanhado por um fluxo de prótons para o espaço intermembrana, que produz um gradiente químico e elétrico e que retorna pela ATP sintase, produzindo a força necessária para a síntese de ATP.

funções mitocondriais têm sido implicadas na neuroprogressão e no prejuízo cognitivo, que, conjuntamente com estudos clínicos, têm demonstrado alterações nos parâmetros do EO presentes na fisiopatologia das doenças psiquiátricas (MOYLAN et al., 2013).

Outro ponto importante a ser lembrado é que as células produzem potenciais na membrana plasmática após estímulo, e, da mesma forma, quando não são excitadas, não há mudanças nas cargas iônicas que produzam a despolarização, mas mostram alguma queda de eletronegatividade. Isso é o suficiente para ativar a proliferação de genes da célula e iniciar o processo de fissão mitocondrial (PEREIRA, 2015). Além disso, o fluxo de prótons, que produz um gradiente eletroquímico, é iniciado pela mudança do potencial de membrana mitocondrial (PMM). Estudos mostram que, com o baixo PMM, a mitocôndria pode aumentar a atividade da CTE entre complexos enzimáticos (ANGRIMANI et al., 2015).

\section{Mitocôndria e Estresse Oxidativo}

A produção de RL é um processo fisiológico e constante, mas quando em desequilíbrio, seja aumentando a produção de RL ou reduzindo a atividade de sistemas antioxidantes, pode ser prejudicial e levar ao EO (CEMILE; ÇIGDEM, 2016). Também pode danificar a membrana celular, gerando peroxidação lipídica e alterando o transporte transmembrana (OBEAGU, 2018). Os RLs são moléculas instáveis e altamente reativas com outras moléculas, produzidas pelo metabolismo aeróbico celular e possuem, pelo menos, um elétron desemparelhado na camada mais externa. Os RLs mais comumente relatados são: superóxido $\left(\mathrm{O}_{2} \bullet\right)$, hidroxila $(\mathrm{OH} \bullet)$, óxido nítrico $(\mathrm{NO} \bullet)$, dióxido de nitrogênio $\left(\mathrm{NO}_{2}\right)$, peroxil (ROO-) e peroxil-lipídeo (LOO) (LUSHCHAK, 2015). $\mathrm{O} \mathrm{O}_{2} \bullet-$ é formado a partir da primeira redução de $\mathrm{O}_{2}$ com um elétron e produzido basicamente em mitocôndrias, microssomas e peroxissomas. Possui pouca reatividade em meio aquoso, e 
suas reações podem desencadear a formação de $\mathrm{OH}^{-}$, $\mathrm{OH} \bullet$ e $\mathrm{O}_{2} \bullet-$ e, quando em meio ácido, de $\mathrm{H}_{2} \mathrm{O}_{2} \cdot \mathrm{O} \mathrm{H}_{2} \mathrm{O}_{2}$ é altamente tóxico para as células em razão da sua longa meia-vida e sua capacidade de atravessar as membranas lipídicas, também reagindo com a membrana eritrocitária e proteínas ligadas ao ferro, causando danos às moléculas por meio da geração de novas EROs, como $\mathrm{OH} \bullet$ (MONICZEWSKI et al., 2015). Conforme observado na Figura 2, as membranas plasmáticas são as estruturas celulares mais susceptíveis aos efeitos deletérios dos RLs, uma vez que a peroxidação lipídica altera sua estrutura, reduzindo a seletividade no transporte iônico e a sinalização transmembrana, prejudicando sua permeabilidade e, consequentemente, o transporte celular. Essas alterações podem resultar em morte celular, uma vez que há a liberação de enzimas hidrolíticas dos lisossomos e formação de produtos da lipoperoxidação, como o malondialdeído (MDA), causado principalmente por radicais $\mathrm{OH} \bullet$, e que pode ser mensurado mediante análises de substâncias reativas ao ácido tiobarbitúrico (TBA-RS). Os níveis de MDA são largamente utilizados como marcadores da peroxidação lipídica nos estados de EO (FRANÇA et al., 2013). Também EROs e ERNs apresentam ação sobre o DNA, principalmente o radical $\mathrm{OH} \bullet$, que leva às alterações na expressão gêni$\mathrm{ca}$, propiciando o desenvolvimento de numerosos processos patológicos crônicos, como câncer e doenças neurodegenerativas. A CTE, na membrana mitocondrial interna, sob condições normais, converte 1-5\% de $\mathrm{O}_{2}$ em EROs (GHEZZI; ZEVIANI, 2012). Além disso, a produção de $\mathrm{O}_{2} \bullet$ e outras EROs ocorrem, principalmente, em dois pontos na CTE: no complexo I (NADH) e no complexo III (ubiquinona - citocromo c redutase). O complexo I e o III podem ser danificados pelo radical $\mathrm{OH} \bullet$. Este radical é altamente reativo e pode causar danos irreparáveis ao mtDNA (KAUPPILA; STEWART, 2015). Concentrações aumentadas de radicais superóxidos, que podem ocorrer em razão de danos no mtDNA, contribuem para a geração do EO, diminuindo a síntese de proteínas importantes para o transporte de elétrons (CASTRO et al., 2012). A formação de RL pode ocorrer de maneira enzimática e não enzimática. São exemplos de reações enzimáticas que envolvem a produção de EROs: a cadeia respiratória mitocondrial, a síntese de prostaglandinas e o sistema do citocromo P450 (SALIM, 2014). Reações não enzimáticas incluem a produção de RL por meio da interação do oxigênio com compostos inorgânicos, como radiação ionizante e Oxphos na mitocôndria. A forma oxidada de $\mathrm{NAD}^{+}$na membrana mitocondrial interna é consequência da Oxphos, que é uma das principais fontes de produção de EROs (SHAFIQUE et al., 2017).
Para reduzir os danos causados pela produção de RL, o organismo possui um sistema de defesa antioxidante, que pode ser dividido em enzimático e não enzimático. Antioxidantes não enzimáticos incluem endógenos como glutationa (GSH), que têm a função de remover RLs tóxicos, ou seja, convertê-los em produtos intermediários não tóxicos, e os exógenos, em que a grande maioria deles são obtidos a partir de fontes alimentares, que podem incluir vitaminas C, E ( $\alpha$-tocoferol), carotenoides, compostos organosulfurados, minerais e cofatores que são importante na manutenção da saúde humana (AWAD; ALDOSARI; ABID, 2018). Os antioxidantes enzimáticos são produzidos endogenamente e abrangem um grupo diverso de enzimas que metaboliza RL, repara estruturas celulares ou conjuga diretamente xenobióticos e lipídios peroxidados. Os antioxidantes dessa fase incluem as enzimas superóxido dismutase (SOD), catalase (CAT), glutationa S-transferase (GST), $v$-glutamilcisteína sintetase (GCS), glutationa peroxidase (GSH-Px) e glutationa redutase (GR) (JEEVA et al., 2015). A SOD é a primeira enzima que atua como um componente primário contra as EROs. É responsável por catalisar e dismutar moléculas $\mathrm{O}_{2} \bullet$ em $\mathrm{H}_{2} \mathrm{O}_{2}$ e oxigênio molecular $\left(\mathrm{O}_{2}\right)$ (DAYAL et al., 2017), exercendo uma ação protetora contra danos no DNA. Outras enzimas antioxidantes no sistema endógeno, essenciais para evitar a morte celular, são a CAT e a GSH-Px (KRISHNAMURTHY; WADHWANI, 2012). A CAT é abundante em quase todos os tecidos, localizados principalmente em peroxissomas, catalisando a redução de $\mathrm{H}_{2} \mathrm{O}_{2}$ em $\mathrm{H}_{2} \mathrm{O}$ e $\mathrm{O}_{2}$, reduzindo o dano oxidativo (TODOROVIĆ et al., 2016). $\mathrm{O} \mathrm{H}_{2} \mathrm{O}_{2}$, em baixas concentrações, tende a regular processos fisiológicos como proliferação celular, metabolismo de carboidratos, ativação plaquetária e função mitocondrial. Em altas concentrações, no entanto, causam danos às células. A GSH-Px é uma importante enzima intracelular que desempenha um papel crucial na inibição do processo de peroxidação lipídica e, portanto, protege as células do EO. Da mesma forma que a CAT, ela age catalisando a detoxificação de $\mathrm{H}_{2} \mathrm{O}_{2}$ em $\mathrm{H}_{2} \mathrm{O}$ e $\mathrm{O}_{2}$. Este processo consiste na redução de $\mathrm{H}_{2} \mathrm{O}_{2}$ por GSH na presença de GSH-Px, formando um composto chamado glutationa oxidada (GSSG), que possui uma ponte dissulfeto (UYS; MULHOLLAND; TOWNSEND, 2014). Conforme mostrado na Figura 2, a GSSG é reduzida pela enzima glutationa redutase, usando elétrons derivados da NADPH, que é formada na via das pentoses fosfato sob a ação da enzima glicose-6-fosfato desidrogenase (CASSIA et al., 2018). O SNC é particularmente vulnerável a danos oxidativos porque o encéfalo usa grandes quantidades de oxigênio, produzindo um excesso de EROs, e tem capacidade antioxidante limitada. 
Também no cérebro, os níveis de glutationa são baixos, comparativamente com outros tecidos e/ou células, e estes valores diminuídos podem torná-lo mais sensível a substâncias tóxicas, evidenciando que a glutationa é essencial para o funcionamento do SNC (TODOROVIĆ et al., 2016). Evidências recentes demonstram que a sinalização por EROs tem um papel importante como segundo mensageiro em células imunes inatas e adaptativas. O sistema imune inato responde a padrões moleculares associados a patógenos (PAMPs), ativando receptores de vigilância, como receptores Toll-like (TLRs), receptores semelhantes a NOD (NLRs) e receptores de helicase RIG-like (RLR).
EROs são necessárias para a liberação de citocinas pró-inflamatórias (IL-1 $\beta, \mathrm{TNF} \alpha$, interleucina 8 (IL-8), interferon beta (IFN $\beta$ ) para uma resposta imunológica adequada, controle de patógenos ou reparo de dano tecidual, uma vez que baixos níveis de EROs mantêm um sistema imunológico saudável (MITTAL et al., 2014). Uma diminuição significativa ou aumento nos níveis de EROs, no entanto, inibem ou hiperativam as respostas imunes inflamatórias adequadas, levando a patologias. A exposição crônica às EROs promove diversas alterações na CTE, ocasionando a perda da formação de ATP, produzindo graves danos em proteínas, lipídios e DNA, e, com isso, produzindo diversas pato-

Figura 2 - Estresse oxidativo e sistemas antioxidantes

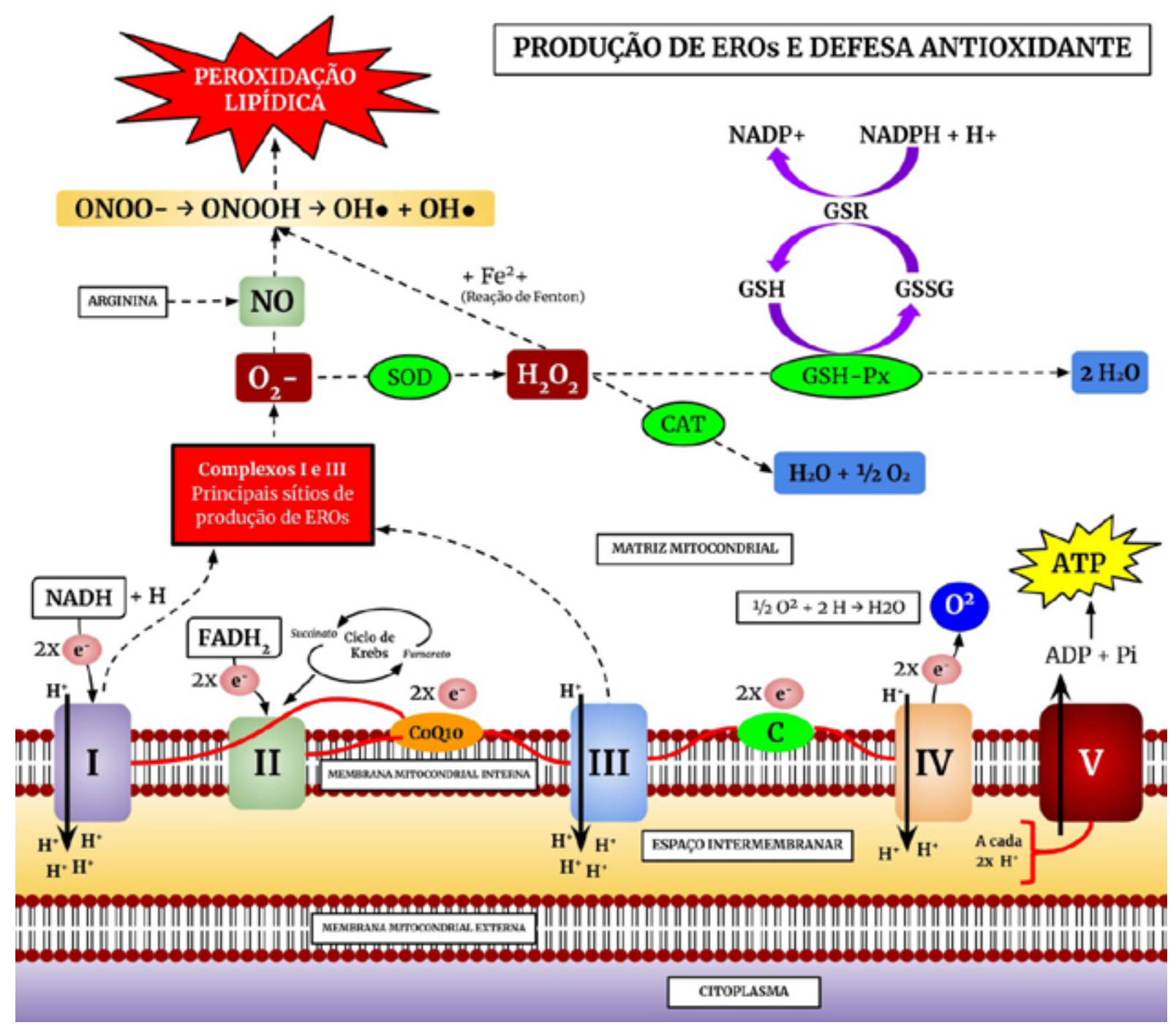

* Sistema de fosforilação oxidativa (OXPHOS) e defesa antioxidante enzimática . Os elétrons derivados do metabolismo celular atingem o complexo I ou II por meio de $\mathrm{NADH}$ ou $\mathrm{FADH}_{2}$, respectivamente. Esses elétrons são, então, transferidos para a coenzima $\mathrm{Q}$ (ubiquinona), uma transportadora de elétrons do complexo I ou II, para III. Neste último, os elétrons são deslocados do citocromo b para o citocromo c e consequentemente transferidos para o Complexo IV (citocromo C oxidase), quando reduzem o $\mathrm{O}_{2}$. Esses elétrons são transportados por intermédio de complexos de proteínas mitocondriais e são acoplados para enviar prótons para o espaço intermembranar. O gradiente eletroquímico gerado é usado pelo Complexo V para a síntese de ATP. Os complexos I e II são responsáveis pela produção do ânion superóxido $(\mathrm{O} 2 \bullet-)$, que é removido pela enzima antioxidante $\mathrm{SOD}$ e, em seguida, produz peróxido de hidrogênio $\left(\mathrm{H}_{2} \mathrm{O}_{2}\right)$, que é removido pelas enzimas CAT e GSH-Px. O superóxido $\left(\mathrm{O}_{2}^{\bullet-)}\right.$ e $\mathrm{H}_{2} \mathrm{O}_{2}$ podem levar à produção de radicais hidroxila $(\mathrm{OH} \bullet$ ), causando lipoperoxidação e lesão celular. 
logias psiquiátricas dentre elas o TB (SCOLA et al., 2016). Para finalizar, este processo foi demonstrado em um importante estudo realizado por Wollenhaupt-Aguiar et al. (2016), que observaram que alterações bioquímicas do soro de pacientes com TB, tanto nos estágios iniciais quanto nos tardios, induziram uma neurotoxicidade em cultura de células neuronais $\mathrm{SH}$ -SY5Y. Foi observada uma diminuição da viabilidade celular com forte redução da densidade de neuritos, principalmente nos estágios tardios da doença.

\section{Estresse Oxidativo e Transtorno Bipolar}

Como já descrito anteriormente, o SNC é particularmente vulnerável ao $\mathrm{EO}$, isso em razão do elevado consumo de oxigênio e, consequentemente, da geração de RL, e também por causa da capacidade antioxidante relativamente baixa desta estrutura. Níveis oxidativos neuronais aumentados podem ter efeitos deletérios na transdução de sinal, plasticidade e resiliência celular (OLMEZ; OZYURT, 2012). Diferentes estudos sugerem que a disfunção mitocondrial desempenha um papel importante na fisiopatologia do TB. Estes estudos mostraram que o aumento do EO serve para sinalizar a disfunção mitocondrial, produzindo uma diminuição na atividade da CTE, podendo ser mensurado tanto no sangue periférico quanto em cérebros post mortem de pacientes com TB (DE SOUSA et al., 2014). Desta forma, o CK é crítico para a atividade mitocondrial, pois produz substratos que alimentam a Oxphos na CTE mitocondrial. Apesar, entretanto, da importância, poucos estudos foram realizados para avaliar a atividade destas enzimas do CK em pacientes com TB. Um destes estudos foi realizado com 18 pacientes com TB em depressão em fase inicial da doença e virgens de tratamento (DE SOUSA et al., 2015). Observou-se que não houve alteração na atividade enzimática no CK em comparação aos controles, provavelmente em virtude dos mecanismos compensatórios que impediram, nas fases iniciais da doença, que elas ocorressem. Não há estudos, entretanto, demonstrando alterações em fases mais tardias da doença. Também é possível que as alterações nas enzimas no CK estejam presentes apenas em fases maníacas, mas não nas fases depressivas, como sugerido em estudo com modelo animal em mania, que apresentou diminuição da atividade das enzimas do CK (FEIER et al., 2013). Da mesma forma, ocorreu diminuição da enzima malato desidrogenase em cérebro post mortem de pacientes com maior tempo de evolução da doença (LEE et al., 2007). O papel do EO na fisiopatologia do TB tem sido investigado em vários estudos que relatam, consistentemente, mudanças nas enzimas antioxidantes, na peroxidação lipídica e nos níveis de NO•, importantes marcadores do EO (TUNÇEL et al., 2015). Os níveis séricos de TBA-RS, durante as fases de mania ou depressão, foram relatados como sendo maiores quando comparados a controles saudáveis (SIWEK et al., 2016). A exposição aguda a EROs levaria ao mau funcionamento dos complexos centrais de elétrons Fe-S na CTE, nos complexos I, II e III, resultando na decomposição da produção de energia mitocondrial (FREDERICO et al., 2012). Além disso, mudanças nas enzimas antioxidantes, CAT, SOD, GSH-Px e peroxidação lipídica, identificada pelo aumento do TBA-RS e danos ao DNA, foram relatadas no TB. No TB ocorre uma diminuição dos níveis de GSH principalmente no córtex pré-frontal dos pacientes (GAWRYLUK et al., 2011). Quanto às enzimas antioxidantes, aumentos séricos foram observados em pacientes tanto em fase maníaca quanto depressiva em relação aos controles saudáveis na maior parte das pesquisas. Halliwell (2006) demonstrou aumento dos níveis de SOD, CAT e GSH-Px em pacientes com TB tanto em fase maníaca quanto depressiva. Salim et al. (2011) também relataram um aumento na atividade do SOD em pacientes medicados ou não com TB durante um episódio maníaco. Outro estudo encontrou um aumento na atividade da SOD em pacientes durante episódios agudos de depressão bipolar, mas não em pacientes eutímicos (SINGH; SINGH; CHAN, 2010). A atividade da catalase também diminuiu em pacientes eutímicos, mas aumentou em pacientes sem medicação durante a mania (RAFFA et al., 2012). Curiosamente, Vasconcelos-Moreno et al. (2017) encontraram a atividade da GSH-Px diminuída em pacientes com TB em comparação com pacientes saudáveis, e demonstraram um aumento de danos no DNA em pacientes com depressão bipolar. Isso demonstra que a gravidade dos sintomas depressivos e maníacos tem uma correlação positiva com os danos no DNA (ANDREAZZA et al., 2010). Em relação à oxidação da proteína mitocondrial, foi demonstrado que os pacientes com TB apresentam níveis mais elevados do que os encontrados nos controles (CHUNG et al., 2013). Também foi observado que existe uma correlação negativa entre a intensidade da depressão e a atividade do complexo II nos linfócitos de pacientes bipolares em depressão, assim como uma correlação positiva entre a depressão e os níveis de SOD, TBA-RS e proteínas carboniladas. Os parâmetros do EO mitocondrial e da atividade do complexo II desempenham papéis importantes no TB (VALVASSORI et al., 2018). Todos estes resultados sugerem que as alterações nas enzimas antioxidantes podem mudar em virtude do tratamento e as fases da doença. As disfunções mitocondriais, a partir 
da ativação das vias inflamatórias, estão presentes no TB. Níveis mais altos de citocinas inflamatórias e diminuição da atividade do complexo I da CTE foram encontrados no cérebro e células periféricas de pacientes com TB, levando ao aumento da produção de EROs mitocondriais (KIM; CHEN; ANDREAZZA, 2015). O mesmo foi encontrado por Kim et al. (2016), mostrando que a ativação do sistema inflamatório, por meio de níveis aumentados de caspase-1, IL-1 $\beta$, IL-6, TNF- $\alpha$, IL-10 e IFN- $\gamma$, induz à produção de EROs, provocando alterações mitocondriais na CTE. Em recente meta-análise exploratória realizada por Rowland et al. (2018), que incluiu 53 estudos, compreendendo 2.467 pacientes com TB e 2.360 controles saudáveis, separados por estado afetivo (eutimia, mania, depressão) e avaliando 14 diferentes biomarcadores para TB, tais como neurotrofinas, marcadores inflamatórios e de EO, observaram que uma combinação de diferentes biomarcadores, como IL-6, BDNF, TNF- $\alpha$ e sTNFR1, apresentavam taxas alteradas de forma diferenciada durante cada estágio de humor. Este resultado pode diferenciar pacientes com TB de controles saudáveis e indicar uma fase específica de humor. Finalmente, o uso de medicações podem interferir no EO. O carbonato de lítio e o ácido valpróico são drogas utilizadas no tratamento do TB, apresentando a estabilização do humor, proporcionando efeitos antioxidantes e protetores para os sintomas do TB, produzindo aumento da função mitocondrial. $O$ lítio aumentou diretamente a atividade do complexo I da CTE mitocondrial (SUN et al., 2006) e ambas as substâncias podem induzir a fosforilação do complexo III e $\mathrm{V}$, aumentando a produção energética (CORENA-McLEOD et al., 2013). Assim, o EO piora a função mitocondrial e a disfunção gera EO, um influenciando o outro. Contudo, os medicamentos atuam melhorando a função mitocondrial como um todo, o que poderia explicar a modulação do humor com o tratamento.

\section{CONCLUSÃO}

Compreender os mecanismos fisiopatológicos e sistêmicos do TB contribui significativamente para melhorar a compreensão da atividade da doença. A disfunção que decorre de múltiplos episódios de humor do TB demonstra evidências fortes que sugerem seu envolvimento com EO e, consequentemente, com os danos da função mitocondrial. A exposição aguda à EROs e ERNs leva a um mau funcionamento dos complexos centrais de elétrons Fe-S na CTE, resultando na queda de produção de energia mitocondrial. Além disso, mudanças nas enzimas antioxidantes CAT, SOD e
GSH-Px, peroxidação lipídica identificada pelo aumento do TBA-RS e danos ao mtDNA, foram relatados nos episódios de depressão e mania bipolar.

Essa revisão contribui para a compreensão deste transtorno complexo e multidimensional, e pode revelar alvos para o diagnóstico, prognóstico e tratamento da doença bipolar.

\section{REFERÊNCIAS}

ALVAREZ-PAGGI, D. et al. Multifunctional cytochrome c: learning new tricks from an old dog. Chem Rev, v. 117, n. 21, p. 13.382-13.460, 2017.

ANDREAZZA, A. C. et al. Mitochondrial complex I activity and oxidative damage to mitochondrial proteins in the prefrontal cortex of patients with bipolar disorder. Arch. Gen. Psychiatry, v. 67, p. 360-368, 2010.

ANGRIMANI, D. S. R. et al. Tools for evaluating the functionality of sperm mitochondria. Rev. Bras. Reprod. Anim., Belo Horizonte, v. 39, n. 2, p. 277-283, 2015.

ASKEW, K. et al. Coupled proliferation and apoptosis maintain the rapid turnover of microglia in the adult brain. Cell reports, v. 18, p. 391-405, 2017.

AWAD, M. A.; ALDOSARI, S. R.; ABID, M. R. Genetic alterations in oxidant and anti-oxidant enzymes in the vascular system. Front Cardiovasc Med, v. 9, p. 105-107, 2018.

BERK, M. et al. Pathways underlying neuroprogression in bipolar disorder: Focus on inflammation, oxidative stress and neurotrophic factors. Neuroscience and Behavioral Reviews, v. 35, p. 804-817, 2011.

BJÖRKHOLM, P. et al. Mitochondrial genomes are retained by selective constraints on protein targeting. Proc Natl Acad Sci USA, v. 112, n. 33, p. 10.154-10.161, 2015.

CASSIA, R. et al. Climate change and the impact of greenhouse gasses: $\mathrm{CO}_{2}$ and NO, friends and foes of plant oxidative stress. Frontiers Plant Sci, v. 9, p. 1-11, 2018.

CASTRO, M. D. R. et al. Aging increases mitochondrial DNA damage and oxidative stress in liver of rhesus monkeys. Exp. Gerontol, v. 47, n. 1, p. 29-37, 2012.

CEMILE, M. S.; ÇIGDEM, E. The effects of oxidative stress and some of the popular antioxidants on reproductive system: A Mini Review. J Nutr Food Sci, v. 6, n. 2, p. 1-3, 2016.

CHEN, Y.; SHENG, Z. Kinesin-1-syntaphilin coupling mediates activity-dependent regulation of axonal mitochondrial transport. Journal of Cell Bio, v. 202, n. 2, p. 351-364, 2013.

$\mathrm{CHU}, \mathrm{C}$. T. Mechanisms of selective autophagy and mitophagy: Implications for neurodegenerative diseases. Neurobiology of Disease, v. 122, p. 23-34, 2019.

CHUNG, C. P. et al. Increased oxidative stress in patients with depression and its relationship to treatment. Psychiatry Res, v. 206, n. 2, p. 213-216, 2013.

CLEMENTE, A. et al. Bipolar disorder prevalence: a systematic review and meta-analysis of the literature. Revista Brasileira de Psiquiatria, São Paulo, v. 37, n. 2, p. 155-161, 2015. 
CORENA-MCLEOD, M. et al. New model of action for mood stabilizers: phosphoproteome from rat pre-frontal cortex synaptoneurosomal preparations. PLoS One v. 8, n. 5, e52147, 2013.

DAYAL, S. et al. Deficiency of superoxide dismutase promotes cerebral vascular hypertrophy and vascular dysfunction in hyperhomocysteinemia. PloS One, v. 12, n. 4, p. 1-15, 2017.

DE SOUSA, R. T. et al. Targeting mitochondrially mediated plasticity to develop improved therapeutics for bipolar disorder. Expert Opin. Ther. Targets, v. 18, p. 1.131-1.147, 2014.

DE SOUSA, R. T. et al. Regulation of leukocyte tricarboxylic acid cycle in drug-naïve Bipolar Disorder. Neurosci Lett, v. 605, p. 65-68, 2015.

DING, W. X. et al. Electron microscopic analysis of a spherical mitochondrial structure. J Biol Chem, v. 7, p. 42.37342.378, 2012.

ENRÍQUEZ, J. A. Supramolecular organization of respiratory complexes. Annu Rev Physiol, v. 78, p. 533-561, 2016.

FEIER, G. et al. Lithium and valproate modulate energy metabolism in an animal model of mania induced by methamphetamine. Pharmacol. Biochem. Behav, v. 103, p. 589-596, 2013.

FERENSZTAJN, E.; RYBAKOWSKI, J. Staging of bipolar affective illness. Psychiatria Polska, v. 46, n. 4, p. 613-626, 2012.

FÖCKING, M. et al. Proteomic analysis of the postsynaptic density implicate synaptic function and energy pathways in bipolar disorder. Translational Psychiatry, v. 6, p. 1-9, 2016.

FRANÇA, B. K. et al. Lipid peroxidation and obesity: Methods to measure the oxidative stress of the obese paciente's plasma. Jornal Português de Gastrenterologia, v. 20, n. 5, p. 199-200, 2013.

FREDERICO, A. et al. Mitochondria, oxidative stress and neurodegeneration. J. Neurol. Sci, v. 322, p. 254-262, 2012.

GAMA, C. S. et al. Staging and neuroprogression in bipolar disorder: a systematic review of the literature. Rev Bras Psiquiatr, v. 35, p. 70-74, 2013.

GAWRYLUK, J. W. et al. Decreased levels of glutathione, the major brain antioxidant, in post-mortem prefrontal cortex from patients with psychiatric disorders. Int. J. Neuropsychopharmacol., v. 14, n. 1, p. 123-130, 2011.

GEISSMANN, F. et al. Unraveling mononuclear phagocyte heterogeneity. Nat Rev Immunol, v. 10, n. 6, p. 453-460, 2010.

GHEZZI, D.; ZEVIANI, M. Assembly factors of human mitochondrial respiratory chain complexes: physiology and pathophysiology. Adv Exp Med Biol, v. 748, p. 65-106, 2012.

GINHOUX, F.; GUILLIAMS, M. Tissue-resident macrophage ontogeny and homeostasis. Immunity, v. 44, p. 439-449, 2016.

GINHOUX, F. et al. New insights into the multidimensional concept of macrophage ontogeny, activation, and function. Nat Immunol, v. 17, p. 34-40, 2016.
GOMEZ, P. E. et al. Tissue-resident macrophages originate from yolk-sac-derived erythro-myeloid progenitors. Nature, v. 518, p. 547-551, 2015.

GRANDE, l. et al. Mediators of allostasis and systemic toxicity in bipolar disorder. Physiol Behav, v. 106, n. 1, p. 46-50, 2012.

GRANDE, I. et al. Staging bipolar disorder: clinical, biochemical, and functional correlates. Acta Psychiatr Scand, v. 129, n. 6, p. 437-444, 2014.

HÄLLBERG, B. M.; LARSSON, N. G. Making proteins in the powerhouse. Cell Metab, v. 20, n. 2, p. 226-240, 2014.

HALLIWELL, B. Reactive species and antioxidants. Redox biology is a fundamental theme of aerobic life. Plant. Physiol, v. 141, p. 312-322, 2006.

HELLWIG, S.; HEINRICH, A.; BIBER, K. The brain's best friend: microglial neurotoxicity revisited. Front. Cell Neurosci, v. 16, p. 7-71, 2013.

HERZ, J. et al. Myeloid cells and their relationship with the central nervous system. Immunity, v. 46, n. 6, p. 943-956, 2017.

HONG, S.; DISSING-OLESEN, L.; STEVENS, B. New insights on the role of microglia in synaptic pruning in health and disease. Current opinion in neurobiology, v. 36, p. 128-134, 2016.

HOPPE, H. C. Mitochondrial calcium channels. Febs Letters, v. 584, n. 10, p. 1.975-1.981, 2010.

IVANNIKOV, M. V.; SUGIMORI, M.; LLINÁS, R. R. Synaptic vesicle exocytosis in hippocampal synaptosomes correlates directly with total mitochondrial volume. J. Mol. Neurosci, v. 49, p. 223-230, 2013.

JEEVA, J. S. et al. Enzymatic antioxidants and its role in oral diseases. J Pharm Bioallied Sci, v. 7, n. 2, p. 331-333, 2015.

JUSTER, R. et al. Sexual orientation and disclosure in relation to psychiatric symptoms, diurnal cortisol, and allostatic load. Psychosomatic Medicine, v. 75, n. 2, p. 103-116, 2013.

KANG, Y.; FIELDEN, L. F.; STOJANOVSKI, D. Mitochondrial protein transport in health and disease. Seminars in Cell \& Developmental Biology, v. 76, p. 142-153, 2018.

KAPCZINSKI, F. et al. Allostatic load in bipolar disorder: implications for pathophysiology and treatment. Neuroscience \& Biobehavioral, v. 32, n. 4, p. 675-692, 2008.

KAUPPILA, J. H.; STEWART, J. B. Mitochondrial DNA: radically free of free-radical driven mutations. Biochimica et Biophysica Acta-Bioenergetics, v. 1.847, n. 11, p. 1.354-1.361, 2015.

KAWABORI, M.; YENARI, M. A. The role of the microglia in acute CNS injury. Metabolic brain disease, v. 30, n. 2, p. 381392, 2014.

KetTEnMANN, H. et al. Physiology of microglia. Physiol. Rev, v. 91, p. 461-553, 2011.

KIM, H. K.; CHEN, W.; ANDREAZZA, A. C. The potential role of the NLRP3 inflammasome as a link between mitochondrial complex I dysfunction and inflammation in bipolar disorder. Neural Plasticity, v. 2.015, p. 1-10, 2015. 
KIM, H. K. et al. Nod-like receptor pyrin containing 3 (NLRP3) in the post mortem frontal cortex from patients with bipolar disorder: A potential mediator between mitochondria and immune-activation. Journal of Psychiatric Research, v. 72, p. 43-50, 2016.

KRISHNAMURTHY, P.; WADHWANI, A. Antioxidant enzymes and human health. Antioxidant enzyme. InTech, 2012. Disponível em: https://www.intechopen.com/books/antioxidant-enzyme/antioxidant-enzymes-and-human-health. Acesso em: 21 jun. 2019.

LEE, B. D. et al. Malic enzyme 2 and susceptibility to psychosis and mania. Psychiatry Res. v. 150, n. 1, p. 1-11, 2007.

LUSHCHAK, V. Free radicals reactive oxygens species, oxidative stress andits classification. Chemico-biological Interactions, v. 224, p. 164-175, 2015.

MACASKILL, A. F.; KITTLER, J. T. Control of mitochondrial transport and localization in neurons. Trends Cell Biol, v. 20, n. 2, p. 102-112, 2010.

MANSUR, R. B.; BRIETZKE, E. The "selfish brain" hypothesis for metabolic abnormalities in bipolar disorder and schizophrenia Trends Psychiatry Psychother, v. 34, n. 3, 2012.

MCEWEN, B. S. Allostasis, allostatic load, and the aging nervous system: role of excitatory amino acids and excitotoxicity. Neurochem Res, v. 25, p. 1.219-1.231, 2000.

MCEWEN, B. S.; GIANAROS, P. J. Stress- and allostasis-induced brain plasticity. Annu.Rev. Med, v. 62, p. 431-445, 2011.

MILENKOVIC, D. et al. The enigma of the respiratory chain supercomplex. Cell Metab, v. 25, p. 765-776, 2017.

MITTAL, M. et al. Reactive oxygen species in inflammation and tissue injury. Antioxid Redox Signal, v. 20, n. 7, p. 1.1261.167, 2014.

MONICZEWSKI, A. et al. Oxidative stress as an etiological factor and a potential treatment target of psychiatric disorders. Part 1. Chemical aspects and biological sources of oxidative stress in the brain. Pharmacologial Reports, v. 67, p. 560-568, 2015.

MOYLAN, S. et al. The neuroprogressive nature of the major depressive disorder: pathways to disease evolution and resistance, and therapeutic implication. Mol Psychiatry, v. 18, n. 5, p. 595-606, 2013.

NAKAGAWA, Y.; CHIBA, K. Review role of microglial M1/ $\mathrm{M} 2$ polarization in relapse and remission of psychiatric disorders and diseases. Pharmaceuticals, v. 7, n. 12, p. 1.0281.048, 2014.

NGUYEN, K. D. et al. Alternatively activated macrophages produce catecholamines to sustain adaptive thermogenesis. Nature, v. 480, p. 104-108, 2011.

OBEAGU, E. I. A review on free radicals and antioxidants. Int. J Curr Res Med Sci, v. 4, n. 2, p. 123-133, 2018.

OLMEZ I.; OZYURT H. Reactive oxygen species and ischemic cerebrovascular disease. Neurochem. Int., v. 60, n. 2, p. 208-212, 2012.

PEI, L.; WALLACE, D. C. Mitochondrial etiology of neuropsychiatric disorders. Biol Psychiatry, v. 83, n. 9, p. 722-730, 2018.
PEREIRA, B. Biogênese mitocondrial e exercício físico: hipótese do acoplamento elétrico-transcripcional Rev Bras Educ Fis Esporte, São Paulo, v. 29, n. 4, p. 687-703, 2015.

PFAFFENSELLER, B. et al. Neurotrophins, inflammation and oxidative stress as illness activity biomarkers in bipolar disorder. Expert Rev Neurother, v. 13, n. 7, p. 827-842, 2013.

PRINZ, M.; PRILLER, J. The role of peripheral immune cells in the CNS in steady state and disease. Nat Neurosci, v. 20, p. 136-144, 2017.

RAFFA, M. et al. Reduced antioxidante defense systems in schizophrenia and bipolar I disorder. Prog. Neuropsychopharmacol. Biol. Psychiatry v. 39, n. 2, p. 371-375, 2012.

ROWLAND, T. et al. Neurotrophins, cytokines, oxidative stress mediators and mood state in bipolar disorder: systematic review and meta-analyses. Br J Psychiatry, v. 213, n. 3, p. 514-525, 2018.

SALIM, S. et al. Novel role of RGS2 in regulation of antioxidant homeostasis in neuronal cells. FEBS Lett, v. 585, n. 9, p. 1.375-1.381, 2011.

SALIM, S. Oxidative stress and psychological disorders. Curr Neuropharmacol, v. 12, n. 2, p. 140-147, 2014.

SCAINI, G. et al. Mitochondrial dysfunction in bipolar disorder: Evidence, pathophysiology and translational implications. Neuroscience and Biobehavioral Reviews, v. 68, p. 694-713, 2016.

SCOLA, G. et al. Lipid peroxidation biomarkers in adolescents with or at high-risk for bipolar disorder. J. Affect. Disord, v. 192, p. 176-183, 2016.

SHAFIQUE, E. et al. Mitochondrial redox plays a critical role in the paradoxical effects of NAPDH oxidase derived ROS on coronary endothelium. Cardiovasc Res, v. 113, p. 234-246, 2017.

SIGITOVA, E. et al. Biological hypotheses and biomarkers of bipolar disorder. Psychiatry and Clinical Neurosciences, v. 71, n. 2, p. 77-103, 2017.

SINGH, V.; SINGH, S. P.; CHAN, K. Review and meta-analysis of usage of ginkgo as an adjunct therapy in chronic schizophrenia. Int. J. Neuropsychopharmacol, v. 13, n. 2, p. 257271, 2010.

SIWEK, M. et al. Thiobarbituric Acid-Reactive Substances: Markers of an Acute Episode and a Late Stage of Bipolar Disorder. Neuropsychobiology, v. 73, p. 116-122, 2016.

SUN, X. et al. Downregulation in components of the mitochondrial electron transport chain in the postmortem frontal cortex of subjects with bipolar disorder. J Psychiatry Neurosci v. 31, p. 189-196, 2006.

SUN, T. et al. Motile axonal mitochondria contribute to the variability of presynaptic strength. Cell Rep, v. 4, n. 3, p. 413-419, 2013.

SYLVIA, L. G. et al. Medical burden in bipolar disorder: findings from the Clinical and Health Outcomes Initiative in Comparative Effectiveness for Bipolar Disorder study. Bipolar Disord, v. 17, n. 2, p. 212-223, 2015.

TODOROVIĆ, N. et al. Olanzapine modulation of hepatic oxidative stress and inflammation in socially isolated rats. Pharmaceutical Sciences, v. 81, p. 94-102, 2016. 
TUNÇEL, Ö. K. et al. Oxidative stress in bipolar and schizophrenia patients. Psychiatry Resarch, v. 228, n. 3, p. 688694, 2015.

UYS, J. D.; MULHOLLAND, P. J.; TOWNSEND, D. M. Glutathione and redox signaling in substance abuse, Biomed. Pharmacother, v. 68, n. 6, p. 799-807, 2014.

VALVASSORI, S. et al. Increased oxidative stress in the mitochondria isolated from lymphocytes of bipolar disorder patients during depressive episodes. Psychiatry Research, v. 264, p. 192-201, 2018.

VASCONCELOS-MORENO, M. P. et al. Telomere Length, Oxidative Stress, Inflammation and BDNF Levels in Siblings of Patients with Bipolar Disorder: Implications for Accelerated Cellular Aging. Int J Neuropsychopharmacol, v. 20, n. 6, p. 445-454, 2017.

VIDYASAGAR, A. What are mitochondria? Live Science. 2015. Disponível em: http://www.livescience.com/50679-mitochondria.html 2015. Acesso em: 21 jun. 2019.

VIETA, E. et al. The clinical implications of cognitive impairment and allostatic load in bipolar disorder. Eur Psychiatry, v. 28, n. 1, p. 21-29, 2013.

WIEDEMANN, N.; PFANNER, N. Mitochondrial machineries for protein import and assembly. Annu Rev Biochem, v. 20, n. 86, p. 685-714, 2017.

WOLLENHAUPT-AGUIAR, B. et al. Reduced Neurite Density in Neuronal Cell Cultures Exposed to Serum of Patients with Bipolar Disorder. International Journal of Neuropsychopharmacology Rapid Communication, v. 19, n. 10, p. 1-5, 2016.

WU, R. et al. The relationship between neurotrophins and bipolar disorder. Expert Rev Neurother, v. 14, p. 51-65, 2014.

ZHANG, C. L. et al. Activity-dependent regulation of mitochondrial motility by calcium and $\mathrm{Na} / \mathrm{K}$-ATPase at nodes of Ranvier of myelinated nerves. J Neurosci, v. 30, p. 3.5553.566, 2010.

ZHU, X.; LEE, B.; CHEN, W. Functional energetic responses and individual variance of the human brain revealed by quantitative imaging of adenosine triphosphate production rates. Journal of Cerebral Blood Flow \& Metabolism, v. 38, n. 6, p. 959-972, 2018. 